\title{
Sciatic Neuropathy in a Dog Secondary to a Bursitis
}

\author{
César Gomes ${ }^{1}$ Angus Anderson ${ }^{2}$ Jennifer Stewart ${ }^{3}$
}

Address for correspondence César Gomes, DVM MRCVS, Queens Veterinary School Hospital, University of Cambridge, Madingley Road, Cambridge, CB3 0ES, United Kingdom of Great Britain and Northern Ireland (e-mail: cg558@cam.ac.uk).

\author{
Abstract \\ Keywords \\ - sciatic \\ - neuropathy \\ - bursa \\ - bursitis \\ - MRI
}

\begin{abstract}
A 4-year-old Dogue de Bordeaux developed acute onset sciatic neuropathy 9 weeks following stifle surgery of the contralateral pelvic limb. Pelvic radiography showed mild bilateral hip dysplasia. Magnetic resonance imaging of the pelvis and lumbosacral spine showed a fluid-filled structure caudal to the hip joint of the affected limb that was causing compression of the sciatic nerve. The lesion was surgically excised and bursitis was confirmed on histological examination. This is the first report of sciatic neuropathy secondary to bursitis in the dog.
\end{abstract}

\section{Introduction}

Neuropathy secondary to bursitis formation has not been reported in the dog but is recognized in humans associated with rheumatoid and osteoarthritis, synovial chondromatosis $^{1}$ and synovial cysts. ${ }^{2}$ Unilateral sciatic neuropathy is encountered in dogs and may occur secondary to pelvic or femoral fractures ${ }^{3,4}$ latrogenic sciatic nerve injury is reported following surgery of the pelvis, ${ }^{4}$ femur ${ }^{5}$ and hip joint $^{6}$ and following injections into the caudal proximal pelvic limb muscles. ${ }^{7}$ Piriformis bursitis has been described as a cause of sciatic neuropathy in humans. ${ }^{8}$

\section{Case History}

A 4-year-old, male neutered, Dogue de Bordeaux was referred for evaluation of left pelvic limb lameness of several months duration. No treatment had been given prior to referral. The dog was diagnosed with cranial cruciate ligament disease of the left stifle and a tibial plateau levelling osteotomy was performed. Recovery was uneventful and at re-examination 6 weeks postoperatively the patient had excellent use of the affected limb. Nine weeks postoperatively, the dog developed acute onset right pelvic limb lameness, and re-presented 1 week subsequently.

received

July 25, 2018

accepted after revision

November 28, 2018

\section{Clinical Findings}

On physical examination, the dog's clinical parameters were within normal limits and body condition score was $5 / 9$ (weight $65 \mathrm{~kg}$ ). Orthopaedic examination revealed a semi-plantigrade stance and occasional knuckling of the foot on the right pelvic limb. Mild gluteal muscle atrophy was observed compared with the contralateral side. Assessment of proprioception in this limb revealed delayed postural reactions. The right patellar reflex showed pseudo-hyperreflexia and the cranial tibial reflex was absent. There was reduced stifle flexion and absent flexion at the level of the hock on withdrawal reflex. Sensation of the toes appeared normal. Localization was consistent with a peripheral sciatic neuropathy. No pain response could be elicited on palpation or manipulation anywhere in the right pelvic limb or lumbosacral spine.

\section{Diagnostic Techniques}

Radiographs of the right stifle showed a mild synovial effusion. Hock and lumbosacral spinal radiographs were normal. A ventrodorsal radiograph of the pelvis showed mild bilateral hip dysplasia. Five days after presentation, magnetic resonance imaging (MRI) (Phillips Achieva Medical Systems 1.5 T, United Kingdom) was performed. Dorsal T2w (weighted) and Short-TI
License terms Stuttgart - New York
DOI https://doi.org/ 10.1055/s-0039-1677750. ISSN 2625-2325. 
inversion recovery (STIR) (repetition time-TR $=3900 \mathrm{~ms}$, echo time-TE $=15 \mathrm{~ms}$, inversion time-TI $=160 \mathrm{~ms})$, T1 $\mathrm{w}$ $(\mathrm{TR}=300-415 \mathrm{~ms} ; \mathrm{TE}=8-11 \mathrm{~ms})$ and T2w $(\mathrm{TR}=5390-6607$ ms; TE $100-110 \mathrm{~ms}$ ) sagittal and transverse and post-contrast (Gadolinium $0.1 \mathrm{mg} / \mathrm{kg}$, Gadovist, Bayer plc) T1w sequences of all three planes of the pelvis and lumbosacral junction were acquired. All images were acquired with a slice thickness of $4 \mathrm{~mm}$ and an inter-slice gap of $4.4 \mathrm{~mm}$ aside from the dorsal sequences (slice thickness $3.5 \mathrm{~mm}$, inter-slice gap of $3.8 \mathrm{~mm}$ ).

Surrounding the right coxofemoral joint and following the contour of the greater trochanter, there was a well-demarcated round to crescent-shaped approximately $2 \times 3 \mathrm{~cm}$ mass lesion. The lesion extended mainly in a dorsal and caudal direction, mildly displacing the gluteal and piriform muscles. Peripherally, this lesion was T1w isointense to muscle ( - Fig. 1) and T2w moderately hyperintense to surrounding muscles. Centrally, the lesion showed a strongly $\mathrm{T} 2 \mathrm{w}$ hyperintense signal and was faintly hypointense to the adjacent muscle. On STIR sequences, the centre of the lesion was strongly hyperintense and its periphery was isointense to adjacent muscles. On post-contrast images (-Fig. 2), the periphery of the lesion had a layered appearance, with a thick inner rim of contrast enhancement surrounded by a thinner outer rim showing no contrast uptake. No contrast enhancement was seen in the centre of the lesion. The lesion was located dorsal to the sciatic nerve and causing subjective compression of the nerve against the ischium, although no contrast enhancement was seen in this nerve. No reactive changes of the surrounding bones or soft tissues were noted. From these imaging characteristics, the mass was determined to be a fluid-filled structure encapsulated by an area of contrast-enhancing vascularized tissue and an outer non-contrast enhancing fibrous capsule.

\section{Diagnosis/Treatment}

Surgical exploration of the tissues caudal to the right hip joint was undertaken by a standard caudodorsal approach. ${ }^{9}$ This

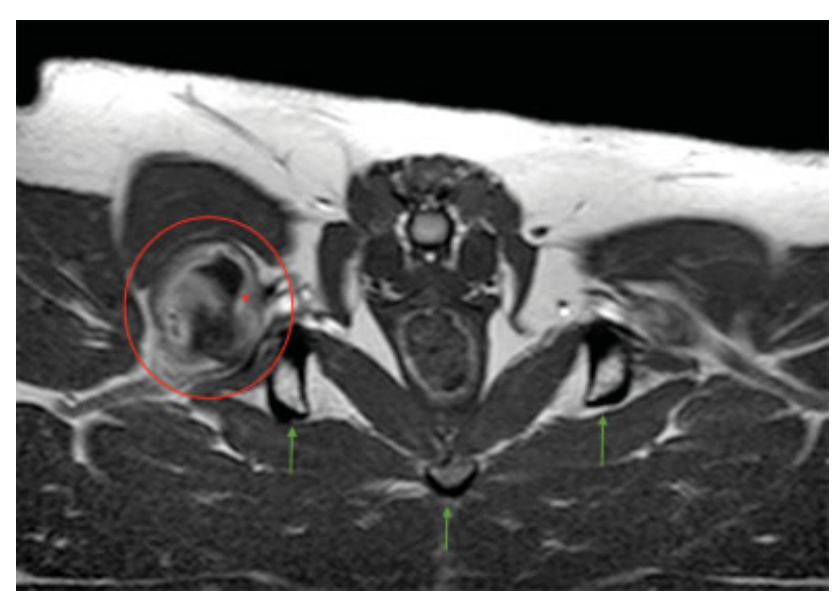

Fig. $1 \mathrm{~T} 1 \mathrm{w}$ (weighted) transverse post-contrast image of the pelvis just caudal to the greater trochanter of the femur. Pubis and pelvis are pointed out by the green arrows. Dorsal is to the top of the image. The lesion is seen on the left side of the patient (red circle) as a centrally T1w hypointense lesions surrounded by a thick contrast enhancing rim (red asterisk) which itself is surrounded by a thin hypointense rim.

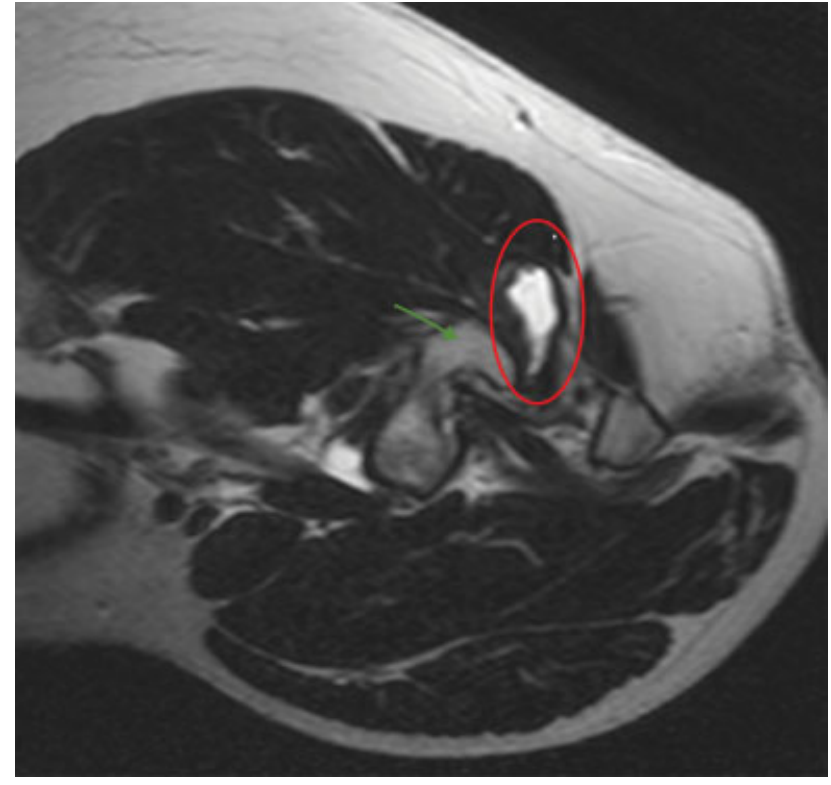

Fig. 2 T2w (weighted) sagittal image of the femur at the level of the greater trochanter (green arrow). The lesion is visible dorsally to the greater trochanter as a strongly T2w hyperintensity (red circle) encapsulated in an outer rim that is $\mathrm{T} 2 \mathrm{w}$ isointense to surrounding muscles.

confirmed the presence of a firm soft tissue structure $(\sim 3 \mathrm{~cm}$ in diameter) in the location described above, causing sciatic nerve compression. It was originating from the tissues underlying the caudal border of the middle gluteal muscle adjacent to the joint capsule of the hip joint. Approximately $10 \mathrm{~mL}$ of straw-coloured fluid of low viscosity was aspirated from the lesion for cytological examination. Careful excision of the lesion was performed and exploration of the cavity of the bursa showed no communication with the hip joint. Surrounding connective tissue was sutured with several mattress sutures of 2-0 polydioxanone (PDS, Ethicon) and surgical closure was otherwise routine. Buprenorphine $0.02 \mathrm{mg} / \mathrm{kg}$ three time a day was administered intravenously for 24 hours following surgery. Oral amoxicillin-clavulanic acid $10 \mathrm{mg} / \mathrm{kg}$ BID (Synulox, Pfizer) was given for 5 days, and meloxicam $0.1 \mathrm{mg} / \mathrm{kg}$ SID (Metacam, Boehringer Ingelheim) for 10 days, postoperatively.

Cytological examination of the fluid showed small numbers of large mononuclear cells characterized by ovoid nuclei with prominent nucleoli and abundant cytoplasm, macrophagelike cells and an absence of neutrophils and other inflammatory cells. Histological examination of the lesion showed it to be composed of predominantly collagenous interstitial tissue, with granulation tissue along one aspect. The granulation tissue bed demonstrated progressive maturation characterized by immature congested capillaries lined by plump endothelial cells progressing toward a defined capillary bed. Small capillaries were frequently oriented perpendicular to the surface. Extravasated erythrocytes (haemorrhages) were multifocally noted in these areas. The maturation of the vascular component was associated with increased deposition of extracellular collagenous matrix and decreased density of activated fibroblasts. On the contralateral aspect, papillary 


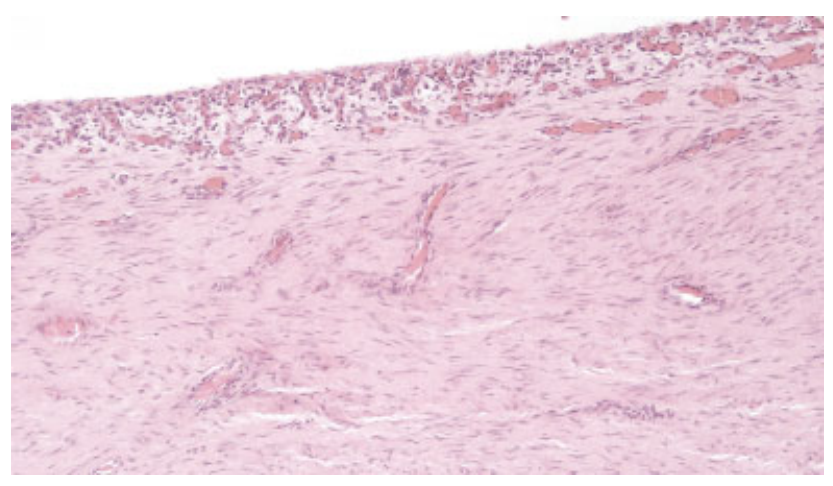

Fig. 3 Histological findings consistent with Bursitis. Haematoxylin and eosin stain (100x).

fronds composed of activated fibroblasts and small capillaries embedded in moderate amount of collagen rarely lined by synovial epithelial cells extended from the surface (-Fig. 3 ). Small numbers of lymphocytes and plasma cells infiltrated perivascular spaces. Histology was consistent with an inflamed bursa-like structure (bursitis).

\section{Progress and Outcome}

The dog was discharged the day after surgery and re-examined 6 weeks later. The semi-plantigrade stance was persistent, but he no longer knuckled over on his toes when walking. Muscle mass of the right pelvic limb had subjectively improved. Hock flexion was still absent on testing of the withdrawal reflex and pseudo-hyperreflexia of the right patella reflex was persistent. Re-examination 12 weeks following surgery showed resolution of the plantigrade stance and mild hock flexion on withdrawal reflex testing. Patella reflex was normal and previous muscle atrophy had almost completely resolved. There was a mild cranial drawer on examination of the right stifle which was not deemed clinically relevant. At last follow-up 6 months postoperatively, the dog was normal.

\section{Discussion}

Bursae are simple connective tissue sacs containing viscous fluid that serve to reduce friction of one tissue over another. Bursae are lined by synovial tissue which produces the lubricating fluid. Most bursae differentiate concurrently with synovial joints during embryogenesis; however, new bursae may develop in response to local stress (such as inflammation or trauma) and pre-existing bursae may hypertrophy. ${ }^{10}$ Preexisting bursae may also develop communications with local joints in response to stress. In the canine hip joint, bursae may be found deep to the tendon of insertion of the deep gluteal muscle, where the internal obturator glides over the lesser ischiatic notch, and deep to the internal obturator and gemelli tendons in the trochanteric fossa between the trochanter major and joint capsule. ${ }^{11}$ Bursitis associated with the piriformis muscle has been reported as a cause of sciatic neuropathy in humans, ${ }^{8}$ but no such bursa appears to be associated with this muscle in the dog.
The dog had a mild joint effusion of the right stifle and mild cranial drawer on examination at 12 weeks of follow-up. This was not considered relevant in this case because no pain or lameness was present and these signs would not explain the neurological deficits seen in this case. It is likely that the dog had early cranial cruciate ligament disease that was not clinically significant at this time. At the last follow-up, the dog was back to normal after conservative management.

Histological appearance of the lesion in this case was more suggestive of bursitis than a synovial cyst, as synovial cells were not identified along the granulation bed. Bursitis is defined as inflammation of the bursa. Repetitive injury within the bursa results in local vasodilatation and increased vascular permeability, stimulating the inflammatory cascade. Bursitis occurs when the synovial lining becomes thickened and produces excessive fluid, leading to localized swelling and pain. ${ }^{12}$ Bursitis has been shown to be painful in humans ${ }^{8}$ and horses but unusually was not the case in this dog. Synovial cysts have been reported in dogs and arise from synovial joints and tendon sheaths. They have a synovial lining but do not always communicate with the joint space. ${ }^{13}$ If they develop from synovial joints along the spine, they may contribute to nerve root compression. ${ }^{14}$ Although rarely reported in dogs, they occasionally develop from joints in the limbs, ${ }^{15}$ but to the authors' knowledge, there are no reports of synovial cysts arising from the hip joint.

It is unclear why bursitis developed in this dog. It is possible that it occurred secondary to increased weightbearing by the limb following contralateral stifle surgery. The large size of this dog may have been a contributory factor. In humans, bursitis has many causes, including autoimmune disorders, crystal deposition (gout and pseudogout), infectious diseases, traumatic events and haemorrhagic disorders, as well as occurring secondary to overuse. ${ }^{10}$ Penetrating trauma as a cause was deemed unlikely in this case given the lack of haemosiderin and/or inflammatory infiltrate. Although small numbers of plasma cells and rare lymphocytes were identified in the granulation tissue bed and small numbers of lymphocytes cuffed vascular spaces in the interstitium, their presence was more consistent with mild chronic irritation or inflammation, supporting the authors' hypothesis that bursitis was stress-induced due to increased weight-bearing in this dog. No communication with the hip joint was observed on MRI or during surgical exploration; however, prior traumatic rupture of the synovial pouch with leakage into the surrounding tissue and subsequent development of a granulation tissue capsule is possible.

In this case, MRI of the pelvis enabled lesion identification. It is also possible that ultrasonography could have enabled a diagnosis; however, ultrasound was unavailable and given the neurological deficits an MRI was opted for. The authors propose that an alternative approach to similar cases may be to drain fluid under ultrasound guidance and if cytological examination is consistent with bursitis, conservative treatment (with rest and non-steroidal anti-inflammatories) may be considered. Surgical excision could then be pursued if the lesion was to recur. In this instance, a decision was taken to excise the bursa to allow the nature of the 
pathology to be fully characterized given how unusual the clinical and imaging findings were.

In conclusion, bursitis caudal to the hip joint should be considered as a differential diagnosis for unilateral sciatic neuropathy in the dog. Surgical excision leads to successful recovery in this case.

\section{Author Contribution}

C. Gomes contributed to data analysis and interpretation.

A. Anderson contributed to acquisition of data and data analysis and interpretation. J. Stewart contributed to acquisition of data. All authors drafted, revised and approved the submitted manuscript.

\section{Conflict of Interest}

None declared.

\section{Acknowledgments}

The authors are grateful to Marie-Aude Genain and Stefano Di Palma for their help in the preparation of this manuscript.

\section{References}

1 Nakano KK. Entrapment neuropathies and related disorders. In: Kelley WN, Harris ED, Ruddy S, Sledge CB, eds. Textbook of Rheumatology, $4^{\text {th }}$ ed. Philadelphia: WB Saunders; 1993:1712-1727

2 Monacelli G, Ceci F, Prezzomoli G, Spagnoli A, Lotito S, Irace S. Posterior interosseus nerve palsy by synovial cyst of proximal radioulnar joint: our experience after 5 years. J Neurosurg Sci 2011;55(02):93-96
3 Gilmore DR. Sciatic nerve injuries in twenty-nine dogs. J Am Anim Hosp Assoc 1984;20:403-407

4 Forterre F, Tomek A, Rytz U, Brunnberg L, Jaggy A, Spreng D. latrogenic sciatic nerve injury in eighteen dogs and nine cats (1997-2006). Vet Surg 2007;36(05):464-471

5 Fanton JW, Blas CE, Withrow SJ. Sciatic nerve injury as a complication of intramedullary pin fixation of femoral fractures. J Am Anim Hosp Assoc 1983;19:687-694

6 Andrews CM, Liska WD, Roberts DJ. Sciatic neurapraxia as a complication in 1000 consecutive canine total hip replacements. Vet Surg 2008;37(03):254-262

7 Rasmussen F. Tissue damage at the injection site after intramuscular injection of drugs. Vet Sci Commun 1978;2:173-182

8 Peh WC, Reinus WR. Piriformis bursitis causing sciatic neuropathy. Skeletal Radiol 1995;24(06):474-476

9 Piermattei DL. Atlas of Surgical Approaches to the Bones and Joints of the Dog and Cat. 3rd ed. Philadelphia: WB Saunders; 1993:248-251

10 Sledge CB. Biology of the joint. In: Kelley WN, Harris ED, Ruddy S, Sledge CB, eds. Textbook of Rheumatology. 4 th ed. Philadelphia: WB Saunders; 1993:1-16

11 Evans HE, De Lahunta A. Millers Anatomy of the Dog. 4th ed. Missouri, United States: Elsevier Saunders; 2013

12 DeLee JC, Drez D. Imaging effusions, cysts, and ganglia. In: DeLee JC, Drez D, Miller MD, eds. DeLee and Drez's Orthopaedic Sports Medicine: Principles and Practice. Philadelphia: WB Saunders; 2003:1646-1648

13 Steiner E, Steinbach LS, Schnarkowski P, Tirman PF, Genant HK. Ganglia and cysts around joints. Radiol Clin North Am 1996;34 (02):395-425, xi-xii

14 Forterre F, Kaiser S, Garner M, et al. Synovial cysts associated with cauda equina syndrome in two dogs. Vet Surg 2006;35(01):30-33

15 Franklin AD, Havlicek M, Krockenberger MB. Stifle synovial cyst in a Labrador Retriever with concurrent cranial cruciate ligament deficiency. Vet Comp Orthop Traumatol 2011;24(02):157-160 